\title{
The challenges of nursing care provision to patients with coronavirus disease 2019
}

\author{
Leila Ebrahimi Sheikh Shabani ${ }^{\circ}$ \\ ${ }^{1}$ Instructor of Medical Surgical Nursing, School of Allied Medical Sciences, Shahrekord University of Medical Sciences, Shahrekord, Iran \\ *Corresponding Author: Leila Ebrahimi Sheikh Shabani, Instructor of Medical Surgical Nursing, School of Allied Medical Sciences, Shahre- \\ kord University of Medical Sciences \\ Received: 11 January 2021, Accepted: 2 June 2021, ePublished: 30 September 2021
}

\section{Dear Editor,}

Coronavirus disease 2019 (COVID-19) is an emerging infectious disease (1) which rapidly turned into a pandemic (2). It has many different physical and mental complications such as respiratory problems, muscular weakness and spasm, general weakness due to prolonged bed rest, worry, anxiety, and insomnia $(3,4)$.

Despite great efforts for COVID-19 prevention and management, the high prevalence of the disease has put heavy pressure on healthcare systems and healthcare providers, particularly nurses. Nursing is a stressful occupation and nurses usually have heavy physical and mental workload. The unknown characteristics of COVID-19, its high prevalence, mortality, and hospitalization rates, and afflicted patients' great need for nursing care services have seriously increased nurses' physical and mental workload and occupational stress and have faced healthcare systems and nurses with different challenges $(5,6)$. Of course, the severity of the challenges depends on individuals' characteristics (5). Examples of these challenges include shortage of staff and equipment, limited applicability of personal protective equipment, poor ventilation in hospital wards, long work hours, prolonged exposure to patients with COVID-19, potential hazards of COVID-19 for staff and their families, mandatory transfer of staff to different hospital wards, limited organizational support, reduced rest time, contradictory rules and regulations, limited quality education about COVID-19, social stigmatization, anxiety, and stress. Evidence shows that 90000 nurses in Iran have so far been affected by COVID-19. The challenges of COVID-19 significantly affect nurses' professional performance and cause them problems such as poor concentration and job burnout (7-9). A study reported that these challenges increased nurses' mental distress by $39.1 \%$, generalized anxiety disorder by $33.73 \%$, and depression among family members by $29.35 \%$. Therefore, accurate identification and management of these challenges and their predictors among nurses and other healthcare providers are essential
(10). Moreover, close adherence to COVID-19 prevention protocols, improvement of people's and healthcare providers' knowledge about the disease, and identification of nurses' concerns and preoccupations are requisite (2) for the effective management of the challenges associated with COVID-19.

\section{Conflict of Interests}

The authors declare no conflict of interests.

Ethical Approval

Not applicable.

References

1. Stübinger J, Schneider L. Epidemiology of coronavirus COVID-19: forecasting the future incidence in different countries. Healthcare (Basel). 2020;8(2):99. doi: 10.3390/ healthcare8020099.

2. Saffari M, Vahedian-Azimi A, Mahmoudi H. Nurses' experiences on self-protection when caring for COVID-19 patients. J Mil Med. 2020;22(6):570-9. doi: 10.30491/ jmm.22.6.570. [Persian].

3. Adhikari SP, Meng S, Wu YJ, Mao YP, Ye RX, Wang QZ, et al. Epidemiology, causes, clinical manifestation and diagnosis, prevention and control of coronavirus disease (COVID-19) during the early outbreak period: a scoping review. Infect Dis Poverty. 2020;9(1):29. doi: 10.1186/s40249-020-00646-x.

4. Brooks SK, Webster RK, Smith LE, Woodland L, Wessely S, Greenberg N, et al. The psychological impact of quarantine and how to reduce it: rapid review of the evidence. Lancet. 2020;395(10227):912-20. doi: 10.1016/s01406736(20)30460-8.

5. Rezazadeh Joudi MK, Rezazadeh Joudi M. The civilizational model of Islam in prophetic tradition with an emphasize on the verses of the Holy Quran. Journal on Quranic Knowledge. 2015;6(20):165-88. doi: 10.22054/rjqk.2015.2357.

6. Shanafelt T, Ripp J, Trockel M. Understanding and addressing sources of anxiety among health care professionals during the COVID-19 pandemic. JAMA. 2020;323(21):2133-4. doi: 10.1001/jama.2020.5893.

7. Nogee D, Tomassoni AJ. COVID-19 and the N95 respirator shortage: closing the gap. Infect Control Hosp Epidemiol. 2020;41(8):958. doi: 10.1017/ice.2020.124.

8. Shrestha GS. COVID-19 pandemic: shortage of personal protective equipment, use of improvised surrogates, and the

( 2021 The Author(s); Published by Shahrekord University of Medical Sciences. This is an open-access article distributed under the terms of the Creative Commons Attribution License (http://creativecommons.org/licenses/by/4.0), which permits unrestricted use, distribution, and reproduction in any medium, provided the original work is properly cited. 
safety of health care workers. J Nepal Health Res Counc. 2020;18(1):150. doi: 10.33314/jnhrc.v18i1.2593.

9. Moayed MS, Mahmoudi H, Ebadi A, Salary MM,

Danial Z. Effect of education on stress of exposure to sharps among nurses in emergency and trauma care wards. Trauma Mon. 2015;20(2):e17709. doi: 10.5812/ traumamon.20(2)2015.17709.

10. Fathi E, Malekshahi Beiranvand F, Hatami Varzaneh A, Nobahari A. Health care workers challenges during coronavirus outbreak: the qualitative study. J Res Behav Sci. 2020;18(2):237-48. doi: 10.29252/rbs.18.2.237

Cite this article as: Ebrahimi Sheikh Shaban L. The challenges of nursing care provision to patients with coronavirus disease 2019. Journal of Multidisciplinary Care. 2021;10(3):93-94. doi: 10.34172/jmdc.2021.18. 